\title{
Finding Shortest Distance Path and Object Detection and Avoidance Using Image Processing and Artificial Intelligence
}

\author{
Omkar A Darekar ${ }^{1}$, Kartik R Kalal ${ }^{2}$, Pratik Holkar ${ }^{3}$, Milap R Disagar ${ }^{4}$ \\ Student, Computer, JSPM's JSCOE, Pune, India ${ }^{1,2,3,4}$
}

\begin{abstract}
In this work we will be implementing Shortest distance path finding algorithm and object detection voidance using Image Processing and artificial intelligence. The work will be focuses on generating shortest path using A star algorithm and object avoidance using Image Processing. Work will also represent insert static map with the help of images. Insertion of object will be testing with different shapes and sizes to represent on the graph.
\end{abstract}

Keywords: Image Processing, Open CV2, Artificial Intelligence, A-Star

\section{INTRODUCTION}

The work presented focuses on the finding shortest path and object detection using image processing and artificial intelligence. Image processing is processing of images in order to improve its quality. The project is divided into two parts: (I) shortest path finding using Static Map (II) Object Detection using machine learning and artificial intelligence. For running object detection we use haar cascade algorithm for face detection .Algorithm developed in python which makes Object detection very user friendly. For shortest path we used the A star algorithm which used heuristic method. For map plotting image processing is done.

(a) Image processing includes

I. Static map image to Gray Scale

II. Thresholding Image

III. Gray Scale to Binary Image

IV. Finding the Contours of image

a. Detection of contours coordinates

b. Plotting points on the graph

- $\quad$ Advantages

- The proposed system does two major tasks simultaneously.

\section{PROBLEM STATEMENT}

In the field of various industries or in research area where environment is not suitable for human being to work In case of blind person, he/she don't not know the path and traverse from one location to another location, he/she may get chance of colliding with object .In case autonomous car driving, we need the shortest distance path which having least obstacle, and in between the detecting obstacles and for avoiding it we need some smart system. For all these cases we need find shortest distance path and object detection and avoidance using image processing and artificial intelligence.

III. PROPOSED SYSTEM

\section{(a) System Architecture}

Proposed system is responsible for finding out shortest path out of all possible path sand and simultaneously detects objects in the path and avoids them. Initially the system is provided with the static map and based on that shortest yet optimal path is evaluated and then object is detected over the path and avoided. The proposed system makes use of one of the best algorithm which is A star algorithm which is used for finding shortest and optimal path. 


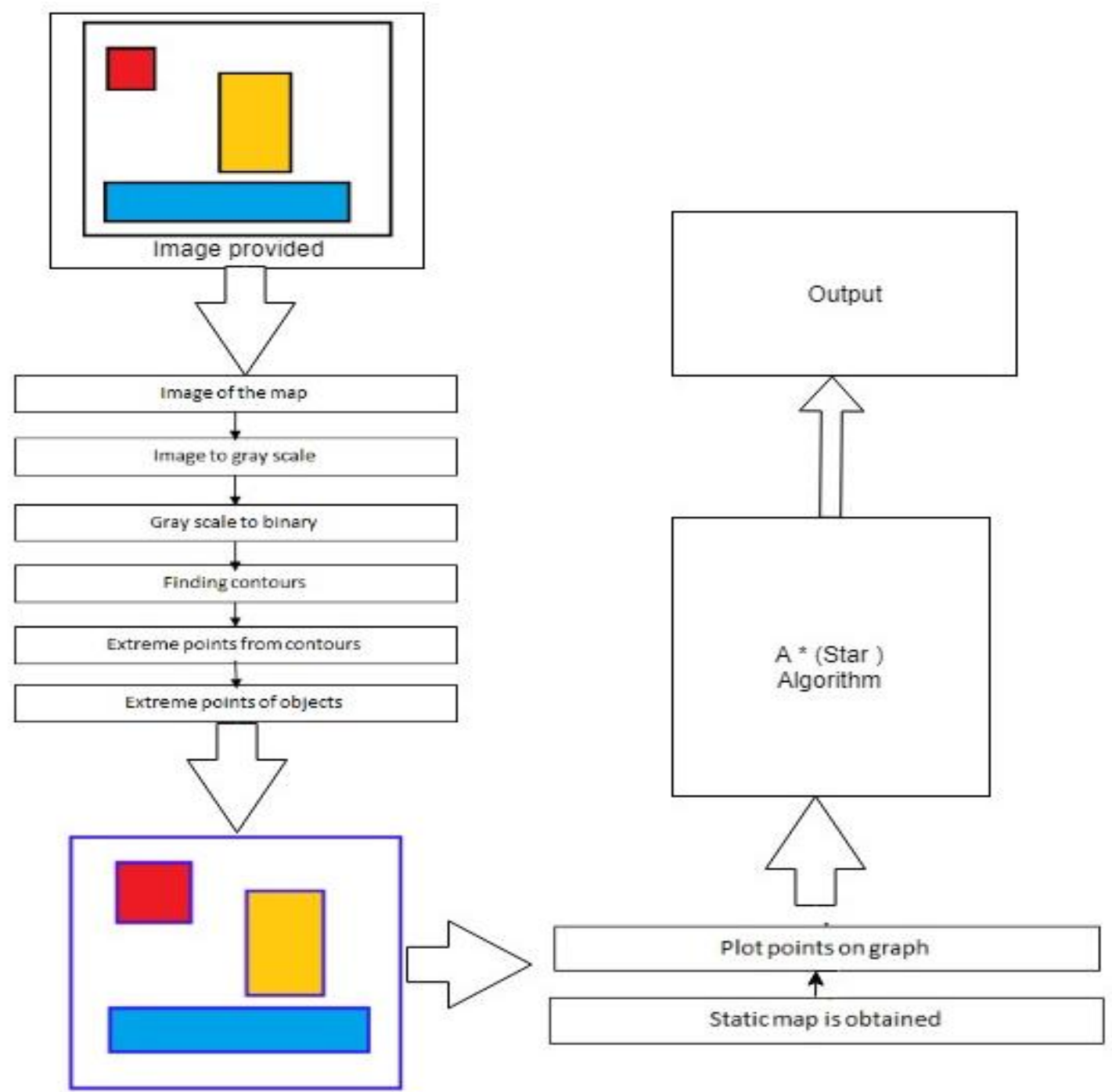

\section{(b) Algorithm Description}

A* is an algorithm that is used in path finding and graph traversal. This algorithm efficiently plots a feasible path between different nodes, on the graph. On a map with many obstacles, path finding from source to destination can be difficult. For instance, a robot without getting much other direction, will continue until it encounters an obstacle, as in the path-finding example as below. However, the $\mathrm{A}^{*}$ algorithm introduces a heuristic approach into a normal graphsearching algorithm, thereby planning ahead at each step so a more optimal decision is made. With $\mathrm{A}^{*}$, a robot would instead find a path in a way similar to the diagram on the right below.

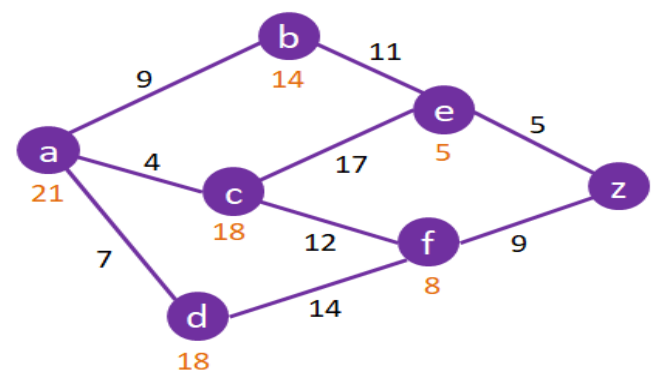

A* works by making a lowest-cost path tree from the initial node to the destination node. For each node, $A^{*}$ uses a function $\mathrm{f}(\mathrm{n})$ that gives an estimate of the total cost of a path using tha thode. Therefore, $\mathrm{A}^{*}$ is a heuristic function is different from an algorithm in thata heuristic is more of an estimate and is not necessarily provably correct. $\mathrm{A}^{*}$ expands paths that are already less expensive by using this function:

$$
\mathbf{f}(\mathbf{n})=\mathbf{g}(\mathbf{n})+\mathbf{h}(\mathbf{n})
$$


where

$\mathrm{f}(\mathrm{n})=$ total estimated cost of path through node $n$

$\mathrm{g}(\mathrm{n})=$ cost so far to reach node $\mathrm{n}$

$\mathrm{h}(\mathrm{n})=$ estimated cost from $\mathrm{n}$ to goal.

(c) Image processing

I. Static Obstacle

a. We gave an image(example - Star shape image)

II. Normal to Gray

a. For shortest path finding initially we are providing an image of static map

III. Gray to Binary

a. It will be converted into Gray scale image

IV. Coutours

a. It will converted binary image to contours for proper border detection.

V. Points from Image

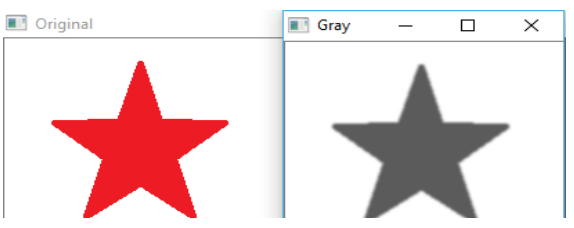

Gray

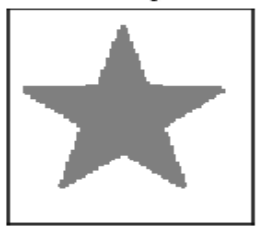

Original

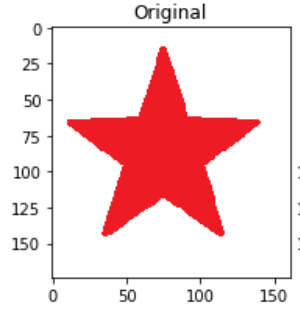

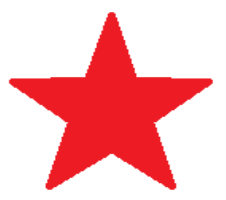

Binary

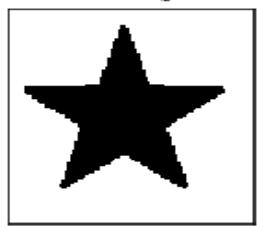

Contours

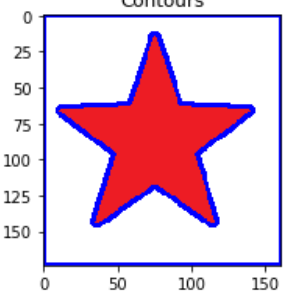

a. After converting original image into binary image the contour of an image is plotted

VI. Points on Graph

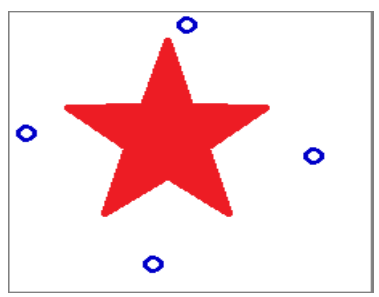

>>

Points of Contours :-

(89.226326, 188.72067)

$(10.238327,90.775543)$

$(110.64628,9.8013916)$

$(189.63428,107.74652)$

$\gg$ |

a. After that we have taken coordinates of that counter and this is how the image is converted in the coordinate format

b. That coordinate plot on the graph and join there vertices to form a obstacle

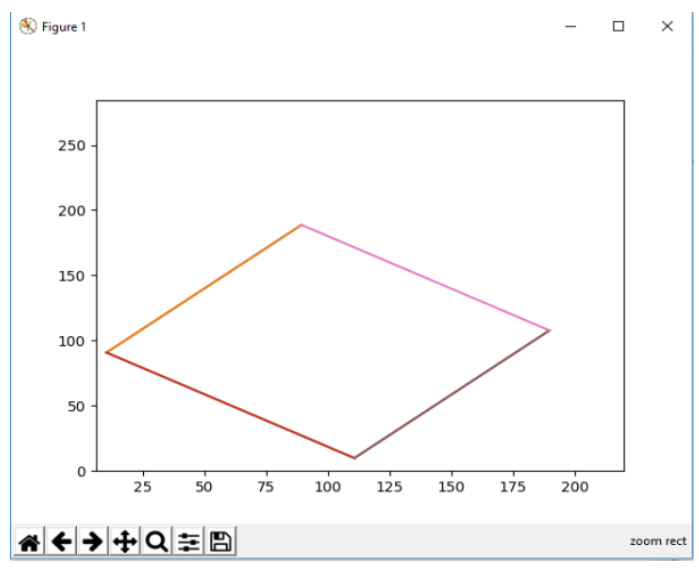




\section{IJARCCE}

Vol. 8, Issue 2, February 2019

VII. A* Algorithm

a. Informally speaking, $A^{*}$ Search algorithms, unlike other traversal techniques, it has "brains". What it means is that it is really a smart algorithm which separates it from the other conventional algorithms. This fact is cleared in detail in below sections

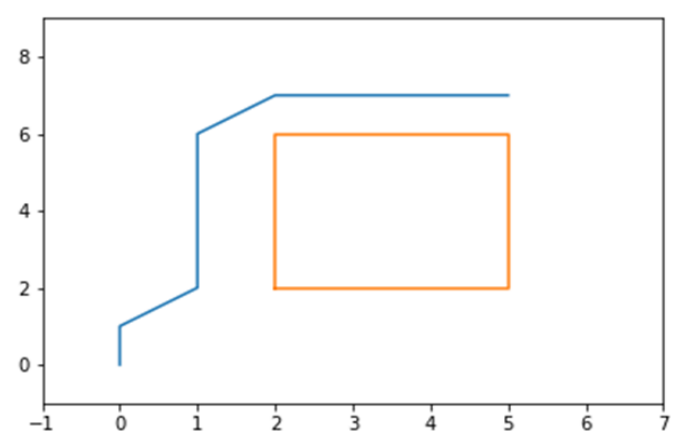

\section{ANALYSIS MODEL}

(a) Flow of project

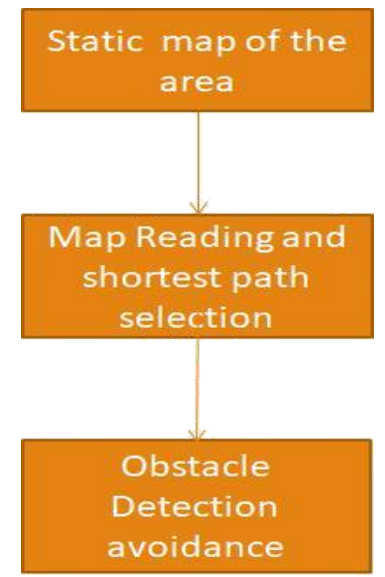

V. UML DIAGRAM

(a) Use case diagram

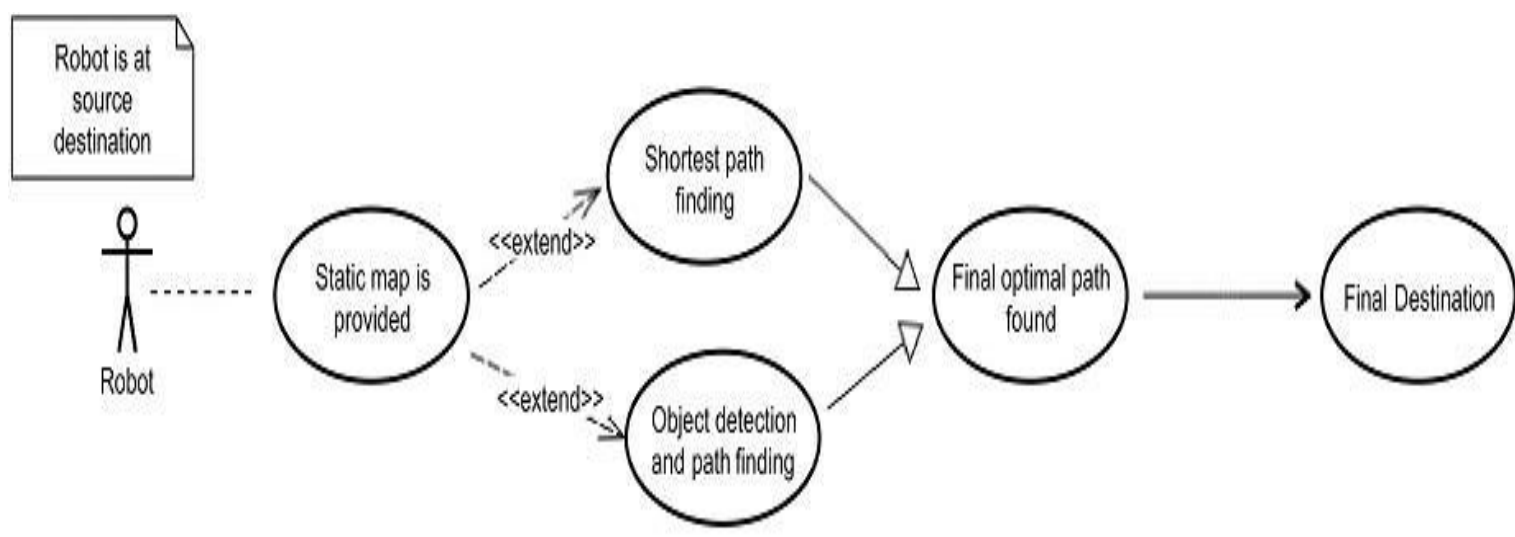

VI. APPLICATION

(a) For guiding blind people to follow unknown path.

(b) Driverless cars

(c) Hazardous areas 


\section{IJARCCE}

Vol. 8, Issue 2, February 2019

\section{CONCLUSION}

we conclude that by using A star algorithm we can find shortest distance path accurately and precisely. From paper no 3 and paper no 4 result we were able to find the knowledge about object detection and avoidance using image processing. In our project we are combining the results of all the above paper into a single system. Till now we are able to merge completely two different operations into our project work. A successful simulation is performed on the shortest path and obstacle detection and avoidance using machine learning and image processing.

\section{REFERENCES}

[1]. Abraham and D. Delling. A hub-based labeling algorithm for shortest paths in road net-works. Experimental Algorithms, 2011.

[2]. A. Bernstein. Maintaining shortest paths under deletions in weighted directed graphs. STOC,page 725, 2013.

[3]. T. M. Chan. All-pairs shortest paths for unweighted undirected graphs in o(mn) time. Proceed-ings of the seventeenth annual ACM-SIAM symposium on Discrete algorithm, pages 514523,2006. (PDF) A Survey of Shortest-Path Algorithms. [accessed Aug 14 2018].

[4]. Bellman R. E. (1958) On a Routing Problem. In Journal Quarterly of Applied Mathematics 16, 87-90

[5]. Qiujin Wu Joanna Hartely., Using K-Shortest Paths Algorithms To Accommodate User Preferences In The Optimization of Public Transport Travel.

[6]. M.C. ChinnaiahDept ECE, Padmasri Dr B.V. Raju Institute of Technology, Medak (Dist), A.P, India INSPEC Accession Number: 14384494 DOI: 10.1109/ICAEE.2014.6838555 Pub- lisher: IEEE Conference Location: Vellore, India 